\title{
CHARACTER THEORY OF FINITE GROUPS WITH TRIVIAL INTERSECTION SUBSETS
}

\author{
JOHN H. WALTER ${ }^{11}$
}

To the memory of TADASI NAKayama

This paper arose out of an effort to present a result which simplifies the application of the theory of blocks to what is often called the theory of exceptional characters. In order to obtain the most effective use of this result, it is necessary to reformulate part of this theory. Thus we present a development which explains an application of $\mathrm{R}$. Brauer's main theorem on generalized decomposition numbers. In particular, we improve a result of D. Gorenstein and the author [8; Proposition 25]. These results are needed in a forthcoming paper and will simplify somewhat the use of this theory in existing papers. We are interested principally in determining the values of certain irreducible characters on trivial intersection subsets. The organization of the theory presented here is influenced by an exposition of M. Suzuki [13] and uses concepts introduced by W. Feit and J. G. Thompson [7]. Also it is hoped that this exposition will serve as an introduction to the theory.

1. Trivial intersection subsets. Let $\mathscr{S}_{\mathfrak{S}}$ be a finite group. Denote by $\mathscr{I} \mathfrak{G}$ the set of complex irreducible characters of $\mathfrak{\xi}$. Let $\mathscr{F} \mathfrak{g}$ denote the module of generalized characters generated $\mathscr{I}_{\mathfrak{5}}$. Let $\mathscr{C}_{\mathfrak{5}}$ denote the vector space of complex valued class functions of $\mathfrak{5} ; \mathscr{C}_{\mathfrak{S}}$ may also be regarded as the dual space of the class algebra (center of the group algebra). Then $\mathscr{F} \mathfrak{F}$ is a basis for both $\mathscr{F} \mathfrak{g}$ and $\mathscr{C}_{\mathfrak{S}}$. Given a subset $\mathscr{S}$ of $\mathscr{I} \mathfrak{g}$, denote by $\mathscr{F} \mathfrak{\mathscr { S }}(\mathscr{S})$ and $\mathscr{C}_{\mathfrak{S}}(\mathscr{S})$, respectively, the submodule of $\mathscr{F} \mathfrak{g}$ and the subspace of $\mathscr{C}_{\mathfrak{g}}$ generated by $\mathscr{S}$. Given a subset $\mathscr{A}$ of $\mathscr{C}_{\mathfrak{S}}$, denote by $\mathscr{I} \mathfrak{S}(\mathscr{A})$ the smallest subset $\mathscr{I}$ of $\mathscr{I}_{\mathfrak{S}}$ such that $\mathscr{C}_{\mathfrak{g}}(\mathscr{S}) \supseteq \mathscr{A}$. Suppose that $\mathscr{D}$ is a normal subset of $\mathscr{S}$. Designate by $\mathscr{C}_{\mathfrak{S}}(\mathfrak{D})$ the subspace of class functions vanishing on $\mathscr{H}-\mathscr{D}$ and by $\mathscr{F} \mathfrak{F}(\mathfrak{D})$ the submodule of generalized characters vanishing on $\mathfrak{Z}-\mathfrak{D}$. For

Received May 17, 1965.

1) This research was supported by the National Science Foundation. 
$\mathscr{S} \subseteq \mathscr{F}_{\mathfrak{S}}$, set $\mathscr{C}_{\mathfrak{S}}(\mathfrak{D}, \mathscr{S})$ to be the subspace of $\mathscr{C}_{\mathfrak{S}}(\mathfrak{D})$ generated by those elements which are linear combinations of elements of $\mathscr{S}$.

Lemma 1. Let $\mathfrak{D}$ be a normal subset of a group $\mathfrak{S}$. Let $d$ be the number of classes of $\mathfrak{S}$ in $\mathfrak{D}$. Then $\operatorname{dim} \mathscr{C}_{\mathfrak{S}}(\mathfrak{D})=d$.

Proof. The proof follows immediately from the observation that $\mathscr{C}_{\mathfrak{g}}(\mathfrak{D})$ is the subspace of linear functions on the class algebra vanishing on the subspace generated by the classes in $\mathscr{S}-\mathfrak{D}$.

For $\lambda$ and $\mu$ in $\mathscr{C}_{\mathfrak{5}}$, set $(\lambda, \mu)_{\mathfrak{g}}$ to be the inner product

$$
(\lambda, \mu)_{\mathfrak{g}}=\frac{1}{|\mathfrak{S}|} \sum_{H \in \mathfrak{g}} \lambda(H) \overline{\mu(H)} .
$$

In particular, define $(\lambda, \lambda)_{\mathfrak{g}}$ to be the weight of $\lambda$. For $\mathscr{C} \subseteq \mathscr{C}_{\mathfrak{g}}$, define $\mathscr{C}^{\perp}=$ $\left\{\lambda \in \mathscr{C}_{\mathfrak{g}} \mid(\lambda, \mu)_{\mathfrak{S}}=0\right.$ for all $\left.\mu \in \mathscr{C}\right\}$.

Lemma 2. Let $\mathfrak{D}$ be a normal subset of $\mathfrak{S}$. Then $\mathscr{C}_{\mathfrak{g}}(\mathfrak{g}-\mathfrak{D})=\mathscr{C}_{\mathfrak{g}}(\mathfrak{D}) \perp$.

Proof. By (1), $\mathscr{C}_{\mathfrak{g}}(\mathfrak{g}-\mathfrak{D}) \subseteq \mathscr{C}_{\mathfrak{5}}(\mathfrak{D})^{\perp}$. By Lemma 1 and the nondegeneracy of (1), $\operatorname{dim} \mathscr{C}_{\mathfrak{S}}(\mathfrak{S}-\mathfrak{D})=\operatorname{dim} \mathscr{C}_{\mathfrak{g}}(\mathfrak{D}) \perp$. Thus the lemma follows.

Let now $\mathbb{S}$ be a group containing $\mathscr{S}$. Let $\lambda \rightarrow \lambda^{*}$ be the induction mapping of $\mathscr{C}_{\mathfrak{S}}$ into $\mathscr{C}_{\mathbb{S}}$ defined for $G \in \mathbb{S}$ by

$$
\lambda(G)^{*}=\frac{1}{|\mathfrak{S}|} \sum_{x \in \mathbb{S} \lambda}\left(X^{-1} G X\right)
$$

where $\lambda\left(X^{-1} G X\right)=0$ when $X^{-1} G X \in \mathbb{B}-\mathfrak{S}$. If $\mathscr{A}$ is a subset of $\mathscr{C}_{\mathfrak{g}}$, let $\mathscr{A}^{*}$ $=\left\{\lambda^{*} \mid \lambda \in \mathscr{A}\right\}$. In particular, $\mathscr{J}_{\mathfrak{S}}^{*} \subseteq \mathscr{F} \mathbb{S}$ and $\mathscr{C}_{\mathfrak{S}}^{*} \subseteq \mathscr{C}_{\mathfrak{S}}$. A linear mapping $\tau$ of a subspace $\mathscr{C}$ of $\mathscr{C}_{\mathfrak{G}}$ into $\mathscr{C}_{\mathfrak{S}}$ is called an isometry if for $\lambda$ and $\mu$ in $\mathscr{C}$,

$$
\left(\lambda^{\tau}, \mu^{\tau}\right)_{\mathfrak{S}}=(\lambda, \mu)_{\mathfrak{S}} \text {. }
$$

We wish to give conditions when the induction mapping is an isometry. Thus we say that a subset $\mathfrak{D}$ of $\mathscr{B}$ is a trivial intersection $\left(T . I\right.$.) subset if $\mathscr{D} \cap \mathfrak{D}^{G} \neq 1$ implies that $G \in \mathbf{N}_{\mathfrak{S}}(\mathscr{D})$. It is easy to see that this is equivalent to the existence of a subgroup $\mathfrak{S}$ satisfying the following two conditions.

$$
\mathbf{C}_{\mathfrak{S}}(D) \subseteq \mathfrak{S} \text { for all } D \in \mathfrak{D}
$$

$$
\text { If } D^{G} \in \mathfrak{D} \text { for } D \in \mathfrak{D} \text { and } G \in \mathfrak{D} \text { then } D^{G}=D^{H} \text { for some } H \in \mathscr{g} \text {. }
$$

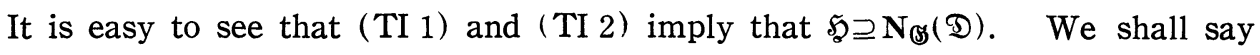
that $\mathfrak{D}$ is a T.I. subset relative to $\mathfrak{S}$. 
Proposition 3. Let $\mathfrak{D}$ be a T.I. subset of $\mathbb{B}$. The induction mapping is an isometry on $\mathscr{C}_{\mathfrak{S}}(\mathfrak{D})$ mapping $\mathscr{F} \mathfrak{F}(\mathfrak{D})$ into $\mathscr{F}$ Fs. Furthermore, for $\theta \in \mathscr{C}_{\mathfrak{S}}(\mathfrak{D})$ and $X \in \mathbb{B}$

$$
\begin{array}{ll}
\theta^{*}(X)=\theta(D) & \text { if } X \text { is conjugate to } D \in \mathscr{D}, \\
\theta^{*}(X)=0 & \text { otherwise. }
\end{array}
$$

Proof. This result is, of course, well-known. Using (TI 1) and (TI 2), equations (4) follow directly from (2), and then (3) follows from (4). That $\mathscr{F}_{\mathfrak{S}}(\mathscr{D})^{*} \subseteq \mathscr{G}(\mathfrak{S}$ is a fundamental property of the induction mapping.

$\mathfrak{S}$ is henceforth assumed to be $\mathbf{N}_{\mathscr{S}}(\mathfrak{D})$ when $\mathfrak{D}$ is a T.I. subset of $\mathbb{B}$.

Denote by $\mathcal{S}^{\mathbb{S}}$ the smallest normal subset containing a subset $\mathcal{S}$ of $\mathbb{S}$.

Lemma 4. Let D be a T.I. subset of $\mathfrak{B}$. Then $\mathscr{C}_{\mathfrak{S}}(\mathfrak{D})^{*}=\mathscr{C}_{\mathfrak{S}}\left(\mathfrak{D}^{\mathfrak{G}}\right)$.

Proof. That $\mathscr{C}_{\mathfrak{S}}(\mathfrak{D})^{*} \subseteq \mathscr{C}_{\mathfrak{S}}\left(\mathfrak{D}^{(\mathfrak{S})}\right.$ ) follows from the previous proposition. (TI 2) implies that the number of classes of $\mathfrak{S}$ contained in $\mathscr{D}$ is the same as the number of classes of $\mathbb{S}$ contained in $\mathfrak{D}^{\mathscr{S}}$. Hence $\operatorname{dim} \mathscr{C}_{\mathfrak{S}}(\mathfrak{D})=\operatorname{dim} \mathscr{C}_{\mathfrak{S}}\left(\mathfrak{D}^{\mathfrak{S}}\right)$. Since the induction mapping is a monomorphism on $\mathscr{C}_{\mathfrak{S}}(D)$, the lemma follows.

2. Coherence and admissibility. Let $\mathscr{D}$ be a T.I. subset of $\mathbb{S}$. Our object is to determine $\left.\Xi\right|_{\mathscr{D}}$ for appropriate $\Xi \in \mathscr{F}(\mathfrak{S}$. We have

$$
\left.\Xi\right|_{D}=\left.\sum_{j=1}^{l} x_{j} \xi_{j}\right|_{\mathscr{D}}
$$

where $\left\{\xi_{j} \mid j=1,2, \ldots, l\right\}=\mathscr{I}_{\mathfrak{g}}$. We shall suppose that the characters $\xi_{j}$ are given so that the problem is to determine the integers $x_{j}$. Of course, when $\mathscr{D} \neq \mathbb{B}$, they are not uniquely determined as $\left\{\xi_{j}|\mathscr{D}| j=1,2, \ldots, l\right\}$ will be dependent.

We use the Frobenius reciprocity formula ${ }^{2)}$

$$
\left(\theta^{*}, \Xi\right)_{\mathfrak{S}}=\left(\theta,\left.\Xi\right|_{\mathfrak{S}}\right)_{\mathfrak{S}}
$$

for $\theta \in \mathscr{C}_{\mathfrak{S}}(D)$ and $\Xi \in \mathscr{I}$ \&s to obtain from Lemma 2 that (5) is equivalent to

$$
\left(\Xi, \theta^{*}\right)_{\mathscr{S}}=\sum_{j=1}^{l} x_{j}\left(\xi_{j}, \theta\right)_{\mathfrak{S}}
$$

for all $\theta \in \mathscr{C}_{\mathfrak{S}}(\mathfrak{D})$. There exists circumstances where (7) can be solved; these

2) Up to this point, one can work as easily with subsets satisfying the two conditions of Dade [4], which were derived from the work of Feit and Thompson [7]. However, in this case, one has to replace the induction mapping by a different isometry, and the Frobenius reciprocity formula has a weaker counterpart (cf. Lemma 9.4 of [7]). Additionai assumptions are needed to distinguish a class of irreducible characters to which the theory can apply. 
will be described by the concepts of coherence and admissibility.

First we say that two subspaces $\mathscr{C}_{1}$ and $\mathscr{C}_{2}$ are separated if $\mathscr{F}_{\mathfrak{S}_{2}}\left(\mathscr{C}_{1}\right) \cap$ $\mathscr{F} \mathscr{F}_{\mathfrak{L}}\left(\mathscr{C}_{2}\right)$ is empty. Given a subspace $\mathscr{C}$ of $\mathscr{C}_{\mathfrak{S}}(\mathfrak{D})$, suppose that

$$
\mathscr{C}=\mathscr{C}_{1} \oplus \mathscr{C}_{2} \oplus \cdots \oplus \mathscr{C}_{k}
$$

where the subspaces $\mathscr{C}_{i}$ are mutually separated. Then a solution to (7) for $\theta \in \mathscr{C}$ is obtained by choosing $x_{j}, j=1,2, \ldots, l$ so that for each $i=1,2$, $\ldots, k$

$$
\left(\Xi, \theta^{*}\right)_{\mathbb{S}}=\sum_{j} x_{j}\left(\xi_{j}, \theta\right)_{\mathfrak{S}}
$$

for all $\theta \in \mathscr{C}_{i}$, the summation $\sum_{j}$ being taken over $\left\{\xi_{j} \mid \xi_{j} \in \mathscr{I}_{\mathscr{S}}\left(\mathscr{C}_{i}\right)\right\}$.

Consider now a subspace $\mathscr{C}$ of $\mathscr{C}_{\mathfrak{S}}(\mathfrak{D})$ and set $\mathscr{S}=\mathscr{F} \mathfrak{F}_{\mathfrak{I}}(\mathscr{C})$. Then we say that $\mathscr{C}$ is coherent (cf. [7] where this concept was introduced) if the induction mapping of $\mathscr{C}$ into $\mathscr{C}_{\mathfrak{S}}$ can be extended to an isometry $\tau$ of $\mathscr{C}_{\mathfrak{S}}(\mathscr{S})$ into $\mathscr{C}_{\mathbb{S}}$ mapping $\mathscr{J} \mathscr{F}_{\mathfrak{S}}(\mathscr{S})$ into $\mathscr{J}\left(\mathfrak{S}\right.$. Then if $\xi_{j} \in \mathscr{S}, \xi_{j}$ has weight 1 ; thus

$$
\xi_{j}^{\tau}=\varepsilon(\tau) \Xi_{j}
$$

where $\varepsilon(\tau)= \pm 1$ and $\Xi_{j} \in \mathscr{I}_{\mathscr{S}}\left(\mathscr{C}^{*}\right)$. Because $\tau$ is a monomorphism, $\tau$ deter mines a bijection of $\mathscr{I}_{\mathfrak{S}}(\mathscr{C})$ onto $\mathscr{F}_{\mathfrak{G}}\left(\mathscr{C}^{*}\right)$ where to $\xi_{j}$ corresponds $\Xi_{j}$.

Suppose then that a separated summand $\mathscr{C}_{i}$ of $(8)$ is coherent. Then if $\Xi=\Xi_{k}$ is in $\mathscr{I}_{\mathbb{S}}\left(\mathscr{C}_{i}^{*}\right)$, a solution for $(9)$ is obtained by setting $x_{k}=\varepsilon\left(\tau_{i}\right)$ and $x_{j}=0$ otherwise; here $\tau_{i}$ is an extension of the induction mapping defined on $\mathscr{S}_{i}=\mathscr{F}_{\mathfrak{S}}\left(\mathscr{C}_{i}\right)$ to an isometry of $\mathscr{C}_{\mathfrak{S}}\left(\mathscr{F}_{i}\right)$. This follows from the observation that $\left(\xi_{j}, \theta\right)=\left(\xi_{j}^{\tau_{i}}, \theta^{*}\right)_{\mathscr{S}}$ for all $\theta \in \mathscr{C}_{i}$ and $\xi_{j} \in \mathscr{S}_{i}$. If each summand $\mathscr{C}_{i}$ of (8) is coherent, then for each irreducible character $\Xi$ of $\mathbb{G}$, there corresponds a uniquely determined $\xi_{i} \in \mathscr{S}_{i}=\mathscr{I}_{\mathfrak{S}}\left(\mathscr{C}_{i}\right)$ provided $\Xi \in \mathscr{F}_{\mathfrak{F}}\left(\mathscr{C}_{i}^{*}\right)$. Then by setting $x_{j}=\varepsilon\left(\tau_{i}\right)$ if $\xi_{j} \in \mathscr{S}_{i}$ is the irreducible character $\xi_{i}$ and setting $x_{j}=0$ if $\xi_{j} \neq \xi_{i}$, it follows from (6) and (9) that with the summation $\Sigma_{i}$ being taken over $\left\{\varepsilon\left(\tau_{i}\right) \xi_{i} \mid \varepsilon\left(\tau_{i}\right) \xi_{i}^{*}=\Xi\right\}$

$$
\eta=\left.\Xi\right|_{H}-\sum_{i \varepsilon}\left(\tau_{i}\right) \xi_{i}
$$

is orthogonal to all $\theta \in \mathscr{C}$ relative to the inner product (1). We say that a subspace $\mathscr{C}$ of $\mathscr{C}_{\mathfrak{F}}(\mathfrak{D})$ is admissible if $\mathscr{C}$ is the direct sum of mutually separated coherent subspaces and if $\mathscr{C}$ is separated from a complement $\mathscr{C}_{0}$ in $\mathscr{C}_{\mathfrak{S}_{2}}(\mathfrak{D})$ and $\mathscr{C}^{*}$ is separated from $\mathscr{C}_{0}^{*}$. 
TheORem 5. Let $\mathscr{C}$ be an admissible subspace of $\mathscr{C}_{\mathfrak{S}}(\mathfrak{D})$. Then with the above notation, it follows for $\Xi \in \mathscr{F}_{\mathbb{F}}\left(\mathscr{C}^{*}\right)$

$$
\Xi\left|D=\sum_{i \varepsilon}\left(\tau_{i}\right) \xi_{i}\right| \mathfrak{D} .
$$

Proof. By Lemma 2, it suffices to show that $(\eta, \theta)_{\mathfrak{S}}=0$ for all $\theta \in \mathscr{C}_{\mathfrak{S}}(D)$. We have shown this if $\theta \in \mathscr{C}$. Our assumption of admissibility implies that if $\theta \in \mathscr{C}_{0},\left(\sum_{i \varepsilon}\left(\tau_{i}\right)_{\tilde{s}_{i}}, \theta\right)_{\mathfrak{S}}=0$ and that $\left(\left.\Xi\right|_{\mathfrak{S}}, \theta\right)_{\mathfrak{H}}=\left(\Xi, \theta^{*}\right)_{\mathfrak{S}}=0$. Thus since $\mathscr{C}_{\mathfrak{S}}(\mathfrak{D})$ $=\mathscr{C} \oplus \mathscr{C}_{0}$, the result follows.

In some cases, particularly in dealing with the case that $\mathscr{2}$ is a Frobenius group, it may not be possible to establish admissibility and to use this theory. But then because of the special structure of $\mathscr{C}$, it is possible to identify $\eta$ in (11). We refer the reader to [6], [7], or [12]. However, following the custom of $[12]$, we call the irreducible characters $\Xi \in \mathscr{F}_{\mathscr{S}}\left(\mathscr{C}^{*}\right)$ exceptional when $\mathscr{C}$ is the sum of mutually separated coherent subspaces.

3. Applications of block theory. An effective way of establishing the existence of admissible subspaces has been to use the theory of blocks. In particular, this was done in [8] and [9] among other places using Proposition 25 of [8], which strongly depends on Brauer's main theorem on generalized decomposition numbers ${ }^{3}$. However, to employ this proposition, it had been necessary in each instance to verify that $\mathscr{C}_{\mathfrak{S}}(\mathfrak{D})$ was a sum of certain mutually separated subspaces. We shall improve upon this by showing that this is always the case.

Let $p$ be a prime. $A$ p-section $\mathbf{S}_{\mathscr{S}}(P)$ of a $p$-element $P$ of $(S$ has been defined to be the set $\left\{\left.P R\left|R \in \mathbf{C}_{\mathscr{S}}(P),\right| R\right|_{p}=1\right\}^{\circledR}$. We shall investigate the situation where a T.I. subset $\mathscr{D}$ of $\mathscr{B}$ is a union of $p$-sections. Let $\mathscr{B}$ be a $p$-block of $\mathscr{S}=\mathrm{N}_{\mathscr{S}}(\mathscr{D})$, and let $\mathscr{B}^{\mathfrak{S}}$ denote the $p$-block of $\mathscr{S}$ corresponding to $\mathscr{B}$ under the Brauer correspondence (cf. [2]). We shall say that two such blocks $\mathscr{B}_{1}$ and $\mathscr{B}_{2}$ are associated if $\mathscr{B}_{1}^{\mathfrak{S}}=\mathscr{B}_{2}^{\mathfrak{S}}$. Associativity is an equivalence relation.

THEOREM 6. Let D be a T.I. subset of a group \& relative to a subgroup $\mathfrak{S}$, and assume that $\mathfrak{D}$ is a union of p-sections of $\mathfrak{S}$. Let $\mathscr{B}$ be a p-block of $\mathfrak{S}$, let

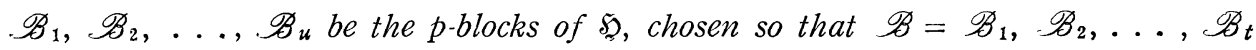
are the p-blocks of $\mathscr{B}$ associated with $\mathscr{B}$. Then

3) Brauer employs his result in a different way (cf. [3]). 


$$
\mathscr{C}_{\mathfrak{S}}(\mathfrak{D})=\oplus_{\mu=1}^{u} \mathscr{C}_{\mathfrak{S}}\left(\mathfrak{D}, \mathscr{B}_{\mu}\right)
$$

and if each $\mathscr{C}_{\mathfrak{S}}\left(\mathfrak{D}, \mathscr{B}_{\mu}\right)$ is coherent,

$$
\mathscr{C}=\oplus_{\mu=1}^{t} \mathscr{C}_{\mathfrak{S}}\left(\mathfrak{D}, \mathscr{B}_{\mu}\right)
$$

is admissible.

Proof. We apply Brauer's theory of generalized decomposition numbers ([1], [2], [10], and [11]). Let $P_{1}, P_{2}, \ldots, P_{s}$ be the representatives of the classes of $p$-elements of $\mathfrak{S}_{\mathcal{B}}$ chosen so that $P_{1}, P_{2}, \ldots, P_{r}$ are representatives of the classes of $p$-elements of $\mathscr{\mathscr { S }}$ contained in $\mathscr{D}$. We have that $\mathscr{g}=\cup_{i=1}^{S} \mathbf{S}_{\mathfrak{g}}\left(P_{\boldsymbol{i}}\right)$ and $\mathfrak{D}=\cup_{i=1}^{r} \mathbf{S}_{\mathfrak{S}}\left(P_{i}\right)$.

Denote by $\mathscr{M} \mathfrak{x}$ the set of Brauer (modular irreducible) characters of a group $\mathfrak{X}$ and by $\mathscr{M}_{\mathfrak{X}}(\mathscr{B})$ those Brauer characters belonging to a block $\mathscr{B}$ of X. Now if $P_{i} R \in \mathbf{S}_{\mathfrak{S}}\left(P_{i}\right)$ and $\xi_{\alpha} \in \mathscr{I}_{\mathfrak{g}}$,

$$
\xi_{\alpha}\left(P_{i} R\right)=\sum_{\beta} d_{\alpha \beta}^{P_{i}} \phi_{\beta}^{P_{i}}(R)
$$

where $\phi_{\beta}^{P_{i}} \in \mathscr{M}_{\mathfrak{F}_{\mathcal{S}}\left(P_{i}\right)}$ and $d_{\alpha \beta}^{P_{i}}$ are the generalized decomposition numbers of $\mathscr{S}_{\mathscr{2}}$. We define for each $\phi_{\beta}^{P_{i}} \in \mathscr{M}_{\mathfrak{C}_{2}\left(P_{i}\right)}, i=1,2, \ldots, s$, class functions $\phi_{\beta}^{P_{i}} \in \mathscr{C}_{\mathfrak{S}}(\mathfrak{D})$ by setting

$$
\begin{array}{ll}
\psi_{\beta}^{P_{i}}(X)=\phi_{\beta}^{P_{i}}(R) & \text { for } X \in \mathbf{S}_{\mathfrak{K}}\left(P_{i}\right) \text { and } X \text { conjugate to } P_{i} R \\
\psi_{\beta}^{P_{i}}(X)=0 & \text { for } X \notin \mathbf{S}_{\mathfrak{S}}\left(P_{i}\right) .
\end{array}
$$

Now let $l\left(P_{i}\right)$ be the number of $p$-regular classes of $\mathbf{C}_{\mathfrak{S}}\left(P_{i}\right)$. This is also the number of $\mathfrak{S}_{\mathscr{S}}$ contained in $\mathbf{S}_{\mathfrak{S}}\left(P_{i}\right)$. Thus if $d$ is the number of classes of $\mathscr{S}$ contained of classes in $\mathfrak{D}, d=\sum_{i=1}^{r} l\left(P_{i}\right)$. On the other hand, there are $l\left(P_{i}\right)$

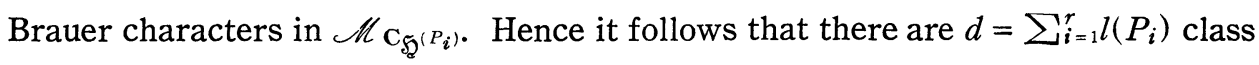
functions $\psi_{\beta}^{P_{i}}$ corresponding to elements $\phi_{\beta}^{P_{i}}$ in $\cup_{i=1}^{r} \mathscr{M} \mathbf{C}_{\mathfrak{F}^{\left(P_{i}\right)}}$. Since the elements

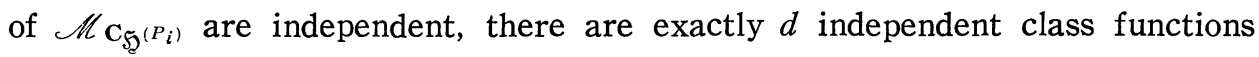
$\psi_{\beta}^{P_{i}}$. We denote by $\mathscr{N}(\mathfrak{D})$ the set of these functions. By Lemma $1, \mathscr{N}(\mathscr{D})$ forms a basis for $\mathscr{C}_{\mathfrak{S}}(\mathfrak{D})$. The same argument also show that there are $l$

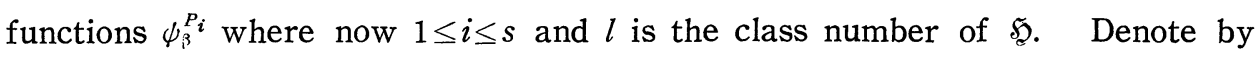
$\mathscr{N}$ this set of functions; then $\mathscr{N}$ forms a basis for $\mathscr{C}_{\mathfrak{5}}$.

Let $\mathscr{B}_{1}, \mathscr{B}_{2}, \ldots, \mathscr{B}_{u}$ be the $p$-blocks of $\mathscr{S}$ where $\mathscr{B}=\mathscr{B}_{1}, \mathscr{B}_{2}, \ldots$, $\mathscr{B}_{t}$ are chosen according to the hypothesis of the theorem. Let $\mathscr{N}(\widetilde{\mathscr{B}}) \mathrm{de}$ signate the set of those $\phi_{\beta}^{P_{i}}$ corresponding to elements $\phi_{\beta}^{P_{i}} \in \mathcal{M} \mathbf{c}_{\mathfrak{S}^{\left(P_{i}\right)}}(\widetilde{\mathscr{B}})$ for 
some $p$-block $\mathscr{\mathscr { B }}$ of $\mathbf{C}_{\mathfrak{F}}\left(P_{\imath}\right)$, and set $\mathscr{N}\left(\mathscr{B}_{\mu}\right)=\cup \mathscr{N}(\widetilde{\mathscr{B}})$ where the union is taken over those blocks $\mathscr{B}$ of $\mathbf{C}_{\mathfrak{S}}\left(P_{i}\right), i=1,2, \ldots, s$ for which $\mathscr{B}^{\mathfrak{D}}=\mathscr{B}_{\mu}$. Set $\mathscr{N}\left(\mathscr{D}, \mathscr{B}_{\mu}\right)=\mathscr{N}(\mathscr{D}) \cap \mathscr{N}\left(\mathscr{B}_{\mu}\right)$. Then $\mathscr{N}=\cup_{\mu=1 \mathcal{N}}^{u}\left(\mathscr{B}_{\mu}\right)$ and $\mathscr{N}(\mathscr{D})=$ $\cup_{\mu=1}^{u} \mathscr{N}\left(\mathfrak{D}, \mathscr{B}_{\mu}\right)$.

Let $\mathscr{K}_{\mathfrak{S}}\left(\mathscr{B}_{\mu}\right)$ and $\mathscr{K}_{\mathfrak{F}}\left(\mathscr{D}, \mathscr{B}_{\mu}\right)$ be the subspaces of $\mathscr{C}_{\mathfrak{S}}$ generated by $\mathscr{A}\left(\mathscr{B}_{\mu}\right)$ and $\mathscr{N}\left(\mathscr{D}, \mathscr{B}_{\mu}\right)$, respectively. Then

$$
\begin{aligned}
& \mathscr{C}_{\mathfrak{S}}=\oplus_{\mu=1}^{u} \mathscr{X}_{\mathfrak{S}}\left(\mathscr{B}_{\mu}\right), \\
& \mathscr{C}_{\mathfrak{S}}(\mathfrak{D})=\oplus_{\mu=1}^{u} \mathscr{K}_{\mathfrak{S}}\left(\mathfrak{D}, \mathscr{B}_{\mu}\right) .
\end{aligned}
$$

We shall establish (13) by showing that

$$
\mathscr{C}_{\mathfrak{S}}\left(\mathfrak{D}, \mathscr{B}_{\mu}\right)=\mathscr{K}_{\mathfrak{S}}\left(\mathfrak{D}, \mathscr{B}_{\mu}\right), \quad \mu=1,2, \ldots, u .
$$

But first we shall establish that $\mathscr{C}_{\mathscr{g}}\left(\mathscr{B}_{\mu}\right)=\mathscr{K} \mathscr{g}\left(\mathscr{B}_{\mu}\right), \mu=1,2, \ldots, u$. Clearly

$$
\mathscr{C}_{\mathfrak{S}}=\oplus_{\mu=1}^{u} \mathscr{C}_{\mathfrak{S}}\left(\mathscr{B}_{\mu}\right)
$$

as $\mathscr{I}_{\mathfrak{h}}=\cup_{\mu=1}^{u} \mathscr{B}_{\mu}$. Hence as (17) and (20) are direct decompositions, it suffices to show that $\mathscr{C}_{\mathfrak{S}}\left(\mathscr{B}_{\mu}\right) \subseteq \mathcal{F}_{\mathfrak{S}}\left(\mathscr{B}_{\mu}\right)$. Therefore, let $\xi_{\alpha} \in \mathscr{B}_{\mu}$. We claim that

$$
\xi_{\alpha}=\sum_{\varangle} d_{x \beta}^{P_{i}} \psi_{\beta}^{P_{i}}
$$

where the summation is over $\psi_{\beta}^{P_{i}} \in \mathscr{N}$. Indeed, every element of $\mathfrak{g}$ is conjugate to an element of the form $P_{j} R$ in $\mathbf{S}_{\mathfrak{S}}\left(P_{j}\right)$. But then $\sum_{\mathcal{r}} d_{\alpha_{\beta}}^{P_{k}} \psi_{\beta}^{P_{i}}(X)=\sum_{\mathcal{r}} d_{\alpha_{\beta}}^{P_{i}} \psi_{\beta}^{P_{i}}\left(P_{j} R\right)$ $=\sum_{\beta} d_{\alpha_{\beta}}^{P_{i}} \phi_{\beta}^{P_{i}}(R)=\xi_{\alpha}\left(P_{i} R\right)=\xi_{\alpha}(X)$ by (16) and (15). Thus (21) holds. But Brauer's main theorem on generalized decomposition numbers states precisely that

$$
\xi_{\alpha}=\sum_{\mathcal{H}\left(\mathscr{B}_{\mu}\right)} d_{\alpha \beta}^{P_{i}} \psi_{\beta}^{P_{i}}
$$

where the summation is over $\psi_{\beta}^{P_{i}} \in \mathscr{A}\left(\mathscr{B}_{\mu}\right)$. Thus $\xi_{\alpha} \in \mathscr{K} \mathscr{H}_{\mathfrak{F}}\left(\mathscr{B}_{\mu}\right)$ and $\mathscr{C}_{\mathfrak{F}_{2}}\left(\mathscr{B}_{\mu}\right)$ $\subseteq \mathscr{K}_{\mathfrak{S}}\left(\mathscr{B}_{\mu}\right)$. Hence $\mathscr{C}_{\mathfrak{S}}\left(\mathscr{B}_{\mu}\right)=\mathscr{K}_{\mathfrak{F}}\left(\mathscr{B}_{\mu}\right)$. Then $\mathscr{K}_{\mathfrak{S}}\left(\mathscr{D}, \mathscr{B}_{\mu}\right) \subseteq \mathscr{C}_{\mathfrak{F}}\left(\mathfrak{D}, \mathscr{B}_{\mu}\right)$. Thus (13) follows.

With the verification (13), we can use Proposition 25 of [8]. Set $\mathscr{D}=$ $\oplus_{\mu=t+1}^{u} \mathscr{C}_{\mathfrak{S}}\left(\mathfrak{D}, \mathscr{B}_{\mu}\right)$. Then $\mathscr{I}_{\mathfrak{H}}(\mathscr{C})=\cup_{\mu=1}^{t} \mathscr{B}_{\mu}$ and $\mathscr{I}_{\mathfrak{S}}(\mathscr{D})=\bigcup_{j=t+1}^{u} \mathscr{B}_{\mu}$. Hence $\mathscr{C}$ and $\mathscr{D}$ are separated. Hence as $\mathscr{C}_{\mathfrak{S}}\left(\mathscr{D}, \mathscr{B}_{\mu}\right)$ are separated. It follows ${ }^{4)}$ from Proposition 25 of [8] that $\mathscr{C}^{*}$ and $\mathscr{L}^{*}$ are also separated. Hence as

4) The argument of Proposition 25 of [8] is correct provided one uses subspaces of class functions rather than modules of generalized characters. The proposition applies to T.I. subsets $\mathscr{D}$ which are the union of $p$-sections. 
$\mathscr{C}_{\mathfrak{S}}\left(\mathfrak{D}, \mathscr{B}_{\mu}\right), \mu=1,2, \ldots, t$, are coherent by assumption, it follows that $\mathscr{C}$ is admissible. This proves the theorem.

In applying Theorem 6 , one determines a basis for each $\mathscr{C}_{\mathfrak{S}}\left(\mathfrak{D}, \mathscr{B}_{\mu}\right), \mu=$ $1,2, \ldots, t$, and using (3) attempts to establish coherence. For this purpose the following proposition is useful.

Proposition 7. Let $\mathfrak{D}$ be a T.I. subset which is a union of p-sections $\mathfrak{D}=$ $\cup_{i=1}^{r} \mathbf{S}_{\mathfrak{S}}\left(P_{i}\right)$ of $\mathfrak{S}=\mathbf{N}_{\mathscr{S}}(\mathfrak{D})$. Let each $\mathbf{C}_{\mathfrak{S}}\left(P_{i}\right), i=1,2, \ldots, r$ contain $m_{i \mu}$ Brauer characters belonging to p-blocks $\widetilde{\mathscr{B}}$ of $\mathbf{C}_{\mathfrak{S}}\left(P_{i}\right)$, such that $\widetilde{\mathscr{B}}^{\mathfrak{K}}=\mathscr{B}_{\mu}$. Then

$$
\operatorname{dim} \mathscr{C}_{\mathfrak{S}}\left(\mathfrak{D}, \mathscr{B}_{\mu}\right)=\sum_{i=1}^{t} m_{i \mu} .
$$

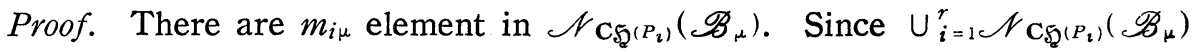
is a basis for $\mathscr{C}_{\mathfrak{S}}\left(\mathfrak{D}, \mathscr{B}_{\mu}\right)$, the proposition follows.

These results are useful in establishing formulas for the orders of groups in terms of the structure of the centralizers of their involutions and the degrees of certain characters. A theorem of Brauer [4] that the principal 2-block $\mathscr{B}_{i}$ in the centralizer of an involution is associate only to itself. Thus one only needs to establish coherence of the subspace $\mathscr{C}_{\mathfrak{F}}\left(\mathscr{D}, \mathscr{B}_{1}\right)$ where $\mathfrak{D}$ is the section $\mathbf{S}_{\mathfrak{S}}(T)$ of an involution $T$ in $\mathscr{S}=\mathbf{C}_{\mathfrak{S}}(T)$ since then $\mathscr{D}$ will be a T.I. subset. In this case, Suzuki's formula $\left(^{* *}\right)$ of [13] can be used directly.

The following proposition allows us to identify the $p$-blocks of $\mathbb{B}$.

Proposition 8. Let D be a T.I. subset of a group $(\mathcal{S}$ which is the union of p-sections of $\mathscr{S}=\mathbf{N}_{\mathfrak{S}}(\mathfrak{D})$. Let $\mathscr{B}_{\mu}, \mu=1,2, \ldots, t$ be a set of associated p-blocks of $\mathfrak{B}$ so that $\mathscr{B}^{\mathfrak{S}}=\mathscr{B}_{\mu}^{\mathfrak{S}}$ for $\mu=1,2, \ldots, t$. Then

$$
\mathscr{C}_{\mathfrak{S}}\left(\mathscr{D}^{\mathfrak{S}}, \mathscr{B}^{\mathfrak{S}}\right)=\sum_{\mu=1}^{t} \mathscr{C}_{\mathfrak{S}}\left(\mathscr{D}, \mathscr{B}_{\mu}\right)^{*}
$$

and $\mathscr{F}_{\mathscr{G}}\left(\mathscr{C}_{\mathfrak{S}}\left(\mathscr{C}^{\mathscr{S}}, \mathscr{B}^{\mathfrak{S}}\right)\right)$ consists of those irreducible characters of $\mathscr{B}^{\mathbb{G}}$ not vanishing on $\mathfrak{D}$. If $\mathfrak{D}$ contains a section $\mathbf{S}_{\mathfrak{S}}(P)$ of a p-element $P$ belonging to a defect group $\mathfrak{V}$ of $\mathscr{B}^{\mathfrak{S}}$, then $\mathscr{P}_{\mathfrak{S}}\left(\mathscr{C}_{\mathfrak{S}}\left(\mathscr{D}^{\mathfrak{S}}, \mathscr{B}^{\mathfrak{S}}\right)\right)=\mathscr{B}^{\mathfrak{S}}$. If $\mathfrak{D}$ does not contain $\mathbf{S}_{\mathfrak{S}}(P) \cap \mathfrak{S}$ for any $P \in \mathfrak{B}$, then $\mathscr{I}_{\mathbb{S}}\left(\mathscr{C}_{\mathfrak{S}}\left(\mathfrak{D}^{\mathbb{S}}, \mathscr{B}^{\mathfrak{S}}\right)\right)$ and thus $\mathscr{I}_{\mathfrak{S}}\left(\mathscr{C}_{\mathfrak{S}}\left(\mathfrak{D}, \mathscr{B}_{\mu}\right)\right), \mu=1,2, \ldots, t$, are empty.

Proof. Lemma 4 asserts that $\mathscr{C}_{\mathfrak{5}}(\mathfrak{D})^{*}=\mathscr{C}_{\mathfrak{S}}\left(\mathfrak{D}^{\mathfrak{S}}\right)$. From this, the proof of Proposition 25 of [8] asserts (24) since (13) holds by virtue of Theorem 6. Let now $\Xi$ be an irreducible character of $\mathscr{B}^{\mathscr{S}}$. If $\left.\Xi\right|_{\mathscr{D}}=0$, then (1) shows that $\left(\left.\Xi\right|_{\mathfrak{g}}, \theta\right)_{\mathfrak{g}}=0$ for all $\theta \in \mathscr{C}_{\mathfrak{g}}(\mathfrak{D})$. Thus $(6)$ shows that $(\Xi, \Theta)_{\mathbb{S}}=0$ for all 
CHARACTER THEORY OF FINITE GROUPS WITH TRIVIAL INTERSECTION SUBSETS 523 $\Theta \in \mathscr{C}_{\mathfrak{S}}\left(\mathscr{D}^{(\mathfrak{S}}, \mathscr{B}^{\mathfrak{F}}\right)$. Clearly then $\Xi \notin \mathscr{F}_{\mathscr{S}}\left(\mathscr{C}_{\mathfrak{S}}\left(\mathscr{D}^{\mathfrak{S}}, \dot{\mathscr{B}}^{\mathfrak{S}}\right)\right)$. Suppose then that $\left.\Xi\right|_{D} \neq 0$. Then set $\Xi^{\prime}$ to be the class function which agrees with $\Xi$ on $\mathscr{D}^{\mathscr{G}}$ and vanishes on $\mathscr{B}-\mathscr{D}^{\mathfrak{S}}$. As $\mathscr{C}_{\mathfrak{S}}\left(\mathfrak{D}^{\mathfrak{S}}\right)=\mathscr{C}_{\mathfrak{S}}(\mathfrak{D})^{*}$ by Lemma $4, \mathscr{N}(\mathfrak{D})^{*}$ forms a basis for $\mathscr{C}_{\mathfrak{S}}\left(\mathfrak{D}^{\mathfrak{S}}\right)$. Lemma 2 shows that $\left(\left.\Xi^{\prime}\right|_{\mathfrak{S}}, \psi\right)_{\mathfrak{S}} \neq 0$ for some $\psi \in \mathscr{N}(\mathfrak{D})$. Then $\left(\Xi, \psi^{*}\right)_{\mathfrak{S}}=\left(\Xi^{\prime}, \psi^{*}\right)_{\mathfrak{S}}=\left(\left.\Xi^{\prime}\right|_{\mathfrak{S}}, \psi\right)_{\mathfrak{S}} \neq 0$, and $\Xi \in \mathscr{I}_{\mathscr{S}}\left(\mathscr{C}_{\mathfrak{S}}\left(\mathfrak{D}^{(\mathfrak{S}}\right)\right)$. Then by (13) applied to the T.I. subset $\mathfrak{D}^{\mathbb{S}}$

$$
\mathscr{C}_{\mathfrak{S}}\left(\mathscr{D}^{\mathbb{S}}\right)=\oplus-\mathscr{C}_{\mathfrak{S}}\left(\mathfrak{D}^{\mathbb{S}}, \overline{\mathscr{B}}\right)
$$

where the summation is taken over the $p$-blocks $\overline{\mathscr{B}}$ of $\mathbb{B}$, and (25) now yields that $\Xi \in \mathscr{F}_{\mathfrak{G}}\left(\mathscr{C}_{\mathfrak{S}}\left(\mathscr{D}^{\mathfrak{S}}, \mathscr{B}^{(\mathfrak{S}}\right)\right)$.

To prove the last statements, take $\Xi \in \mathscr{B}^{\mathscr{S}}$ where $\mathscr{B}^{\mathbb{G}}$ has defect $d$ and defect group $\mathfrak{B}$. Let $\mathfrak{p}$ be a prime ideal divisor of $p$ in a splitting field for $\Xi$. From the definition of a defect group, it follows that $\Xi(R) \neq 0(\bmod p)$ for some $p$-regular element $R$ in $\mathbf{C}(\mathfrak{B})$. But then if $P \in \mathfrak{B}, P R \in \mathbf{S}_{\mathscr{S}}(P)$. Since $\Xi(P R) \equiv \Xi(R)(\bmod p)$, the previous statement of this proposition applies to show that $\Xi \in \mathscr{F}_{\mathfrak{S}}\left(\mathscr{C}_{\mathfrak{F}}\left(\mathscr{D}^{\mathfrak{G}}, \mathscr{B}^{\mathscr{S}}\right)\right)$ if $\mathbf{S}_{\mathscr{S}}(P) \cap \mathscr{S} \subseteq \mathscr{D}$. On the other hand, if $\mathscr{D}$ does not contain $\mathbf{S}_{\mathscr{S}}(P) \cap \mathscr{S}$ for any $P \in \mathfrak{B},(7 \mathrm{~A})$ of $[2]$ implies that $\Xi \mid \mathscr{D}=0$ for all $\Xi \in \mathscr{B}^{\mathscr{S}}$. Now the previous statement of the proposition implies that $\mathscr{I}_{\mathscr{F}}\left(\mathscr{C}_{\mathscr{S}}\left(\mathscr{D}^{\mathfrak{G}}, \mathscr{B}^{\mathfrak{\Phi}}\right)\right)$ is empty. Hence also $\mathscr{I}_{\mathfrak{S}}\left(\mathscr{C}_{\mathfrak{S}}\left(\mathscr{D}, \mathscr{B}_{\mu}\right)\right), \mu=1,2, \ldots, t$, is empty, and the proposition is proved.

\section{References}

[1] R. Brauer, On the connection between the ordinary and modular characters of groups of finite order. Ann. of Math 42 (1941), 926-935.

[2] R. Brauer, Zur Darstellungstheorie der Gruppen endlicher Ordnung II, Math. Zeit. 72 (1959).

[ 3 ] R. Brauer, Investigations of groups of even order. Proc. Nat. Ac. Sci., 47 (1961), 18911893.

[4] R. Brauer, Some applications of the theory of blocks of finite groups, I. J. Algebra, 1 (1964), 152-167.

[ 5 ] E. Dade, Lifting group characters, Ann. of Math. 79 (1964), 590-596.

[6] W. Feit, On a class of doubly transitive permutation groups. Ill. J. Math. 4 (1960), 170186.

[ 7 ] W. Feit and J. G. Thompson, Solvability of groups of odd order. Pac. J. Math. 13 (1963), $775-1029$.

[8] D. Gorenstein and J. H. Walter, On finite groups with dihedral Sylow 2-subgroups, Ill. J. Math. 6 (1952), 553-593.

[9] D. Gorenstein and J. H. Walter, The characterization of finite groups with dihedral Sylow 2-subgroups, I. J. Algebra, 2 (1965), 85-151. 
[10] K. Iizuka, On Brauer's theorem on sections in the theory of group characters, Math. Zeit. 75 (1961), 299-304.

[11] H. Nagao, A proof of Brauer's theorem on generalized decomposition numbers, Nagoya Math. J. 22 (1963), 73-79.

[12] M. Suzuki, On finite groups with cyclic Sylow groups all add primes, Am. J. Math. 77 (1955), 657-691.

[13] M. Suzuki, Applications of group characters. Proc. Symp. Pur Math. vol. I (Finite Groups), Providence, 1959, 88-99.

University of Illinois

Urbana, Illinois 